\title{
Implementation of Tactile Sensing for Palpation in Robot-Assisted Minimally Invasive Surgery
}

\author{
Jelizaveta Konstantinova, Allen Jiang, Kaspar Althoefer, Member, IEEE, Prokar Dasgupta, and \\ Thrishantha Nanayakkara, Member, IEEE
}

\begin{abstract}
Robot-assisted Minimally Invasive Surgery (RMIS) made it possible to perform a number of medical manipulations with reduced patient trauma and better accuracy. Various devices, including tactile sensors, have been developed in recent years to enhance the quality of this procedure. The objective of this paper is to review the latest advancements and challenges in the development of tactile sensing devices designed for surgical applications. In particular the focus is on palpation and probing devices that can be potentially used in RMIS. In addition, we explore the aspects that should be taken into account when designing tactile sensors for RMIS, incorporating biological inspiration of tactile sensing, features of manual palpation, requirements of RMIS. We provide an overview of recommendations for the development of tactile sensing devices, especially in the context of RMIS.
\end{abstract}

Index Terms-Force sensors, Minimally Invasive Surgery, Medical Robotics, Tactile sensors

\section{INTRODUCTION}

$\mathrm{T}$ HE sense of touch is an essential component for our perception of the world. It helps us to navigate in darkness and not only gives us the possibility to detect the presence of an object, but also its shape, size, temperature and mechanical surface properties. It is the only perception system, which is distributed throughout the human body and sends a complex set of signals to the brain originating from a multitude of receptors and nerve endings. They are located in muscles, joints, mucosa, and the surface of the skin, and, respond to various inputs, including touch, pressure, temperature, pain, and body orientation in space.

For human beings, the ability to explore tactile properties of an object is subjective and changes with experience [1]. Therefore, in order to recreate a realistic sense of touch in

Manuscript received 2013. This work was supported in part by the Seventh Framework Programme of the European Commission under grant agreement 287728 in the framework of EU project STIFF-FLOP as well as by Guy's and St. Thomas' Charity, Vattikuti Foundation, NIHR Biomedical Research Centre, MRC Centre for Transplantation.

J. Konstantinova, A. Jiang, K. Althoefer and T. Nanayakkara are with the Department of Informatics, King's College London, Strand, London WC2R 2LS, U.K. (phone: +442078482041 e-mail: jelizaveta.zirjakova@kcl.ac.uk; allen.jiang@kcl.ac.uk; kaspar.althoefer@kcl.ac.uk; thrish.antha@kcl.ac.uk).

P. Dasgupta is with the Department of Urology, King's College London, Guy's Hospital, London SE1 9RT, U.K. (e-mail: prokarurol@gmail.com).

Copyright (c) 2013 IEEE. Personal use of this material is permitted. However, permission to use this material for any other purposes must be obtained from the IEEE by sending a request to pubs-permissions@ieee.org. robotic applications, multiple sensing devices and a complex control system for the behavioral control are needed to perceive and interpret different types of stimuli. Nowadays, no sensor system exists that is capable of accurately measuring the full complexity of tactile cues on the same level as the human tactile receptive system. Thus, the development of such technologies is an important technical problem and scientific challenge.

Tactile sensing in robotics covers a wide spectrum of different fields, including surgical and healthcare applications, industrial handling devices, and dexterous robotic hands. The focus of this review paper is on the state of the art of tactile sensors for use in RMIS. The active development and practical implementation of tactile sensors started at the beginning of the 21st century with ground breaking technologies in medicine and healthcare [2]. The review by Lee and Nichols [2] shows that, at the end of the 20th century, more publications on tactile sensors, designed for practical applications, started to appear. They also report on the growing interest from the medical community towards tactile sensing.

Despite its short history, medical tactile sensing has a broad background. The reviews [3]-[5] examine tactile sensor research from the previous decade, and the reader is referred to these papers for an overview of earlier medically-applied tactile sensor work. The main focus of another review paper is on the recent publications, which built on recent research exploring and studying the mechanisms of tactile sensing in humans, which is believed to be an ideal model for artificial touch sensors [6]. In addition, the interested reader is directed to two thorough reviews providing a general overview of tactile technologies [7], [8]. In our paper, along with the overview of tactile devices for RMIS, we will discuss how biology can be used as inspiration for the development of tactile sensors. We will also discuss the important issue of the interaction between soft tissue and medical tools and its impact on tactile sensor requirements, specifically using tissue palpation as an example to understand the desired design characteristics of the sensing devices.

\section{A. Motivation}

Shorter recovery time, reduced pain and trauma, and less medication needed are frequently-referred positive aspects of RMIS [9]. Moreover, there are a number of benefits for the surgeon. For example, the telerobotic da Vinci surgical system by Intuitive Surgical ${ }^{\circledR}$ provides a high resolution, three- 
dimensional view of the operation field. Surgical precision is increased by the ability to filter out natural hand tremor. In addition, the ability to reduce muscle fatigue and ease of use for the surgeon complements the other benefits of the procedure. These are the key reasons for the worldwide popularity and continuous development for minimally invasive techniques [10]. According to studies in [11], [12], RMIS shows its benefits for procedures such as hysterectomy, prostatectomy, and thyroid surgery.

Nevertheless, the use of RMIS is accompanied with a loss of tactile sensations for surgeons. Direct touch access to the organ is not available, and, consequently, organ palpation cannot be conducted. The ability to feel an organ's mechanical properties during the surgery can help to detect tumors and lumps in the organ, as well as to understand the margin of the abnormality to conduct dissection. However, the main feedback RMIS users receive is through vision, and in order to understand tool-tissue interaction forces, surgical experience is required [13]. Visualization during surgery can only partially compensate for the lack of tactile feedback, as vision just provides information about the outer surface of an organ; however at times the abnormality is covered with a layer of healthy tissue. Therefore, the thorough assessment of an organ's mechanical properties should be realized with the help of force sensing devices.

Artificial tactile sensing can be implemented as the mechanical properties of healthy soft tissues differ from the cancerous ones - the elastic modulus of tumors is approximately higher by a factor of ten [14], [15]. The knowledge of the exact tumor location allows surgeons to perform a more accurate surgical removal and to preserve healthy tissue as much as possible. Thus, the trauma of the surgery would be reduced.

Tactile feedback during the surgery, along with the visualization of an operation site, can enhance the dexterity and controllability of a surgical instrument, which can improve the quality of tool and tissue interaction and can help to achieve reduced trauma [16], [17]. Conversely, the lack of tactile feedback during RMIS can cause serious operative, infectious and other complications while performing difficult manipulations, for instance coronary artery bypass grafting [18].

The rest of this paper is organized as follows: part II discusses the requirements for tactile sensing devices for RMIS. Then, a classification and overview of current devices is presented in part III. Finally, the discussion, covering some important details of tactile sensing, is presented in part IV.

\section{TACTILE SENSING IN RMIS - REQUIREMENTS AND CHARACTERISTICS}

In this section, the overview of specific requirements and characteristics of tactile devices for RMIS is presented. As mentioned, the lack of tactile feedback is an important concern for RMIS. However, tactile sensation is not yet broadly implemented for real surgical applications, as described in [4]. One of the main reasons for that may be the strict certification requirements specified for medical devices. Therefore, accuracy and stability of the devices and their measurements is a very important issue. For example, if the sensor is used to detect the presence and location of an abnormality, incorrect tactile information may lead to false tumor detection or may miss detecting an abnormality. Such variability of results will influence the clinical outcome of the examination and subsequent treatment. Many natural factors and processes can also cause variability of tactile data. For instance, even a patient natural breathing causes a displacement of the internal organs. In addition, the surface properties of an organ may vary because of blood and other fluids, which can be excessive during surgery. Thus, the sensing signal can be influenced by changes in friction between the sensing tool and tissue. These variability factors for tactile sensing should be taken into account when developing devices for RMIS.

Keeping surgical procedures as short as possible is important, as any additional surgery time can induce additional complications to the patient and will increase the cost of the procedure - the surgeon does not have the possibility to perform multiple tactile scans of an organ to confirm the presence or location of a tumor inside an organ. A probe for tactile data detection should ensure the possibility to measure soft tissue properties within a short period of time, i.e. operate at fast traversing speeds.

RMIS is performed through small incisions, or trocar ports, whose diameters vary from 5 to $12 \mathrm{~mm}$ [19]. Therefore, all surgical devices should be miniaturized to fit through a trocar port. In addition, tactile sensing instrument should be either sterilizable or disposable. The latter case could be practically realized if low cost components are used. For sterilization it is necessary to take into account that it can be performed in a steam autoclave using high pressure vapor and high temperature [20], in a chemiclave with low humidity and chemical solution [21], or using gas sterilization [22]. The simplicity of the whole design of tactile device is another desirable property leading to low cost and affordability.

In some cases, RMIS is performed using a magnetic resonance imaging (MRI) [23]. That procedure is required when the real time observation of an instrument position relative to internal organ and areas of interests is needed. MRI is enhancing the contrast of soft tissues and can be used for multi-planar spatial visualization [24], [25]. In contrast to Xray computer tomography and fluoroscopy, it is not subject to ionizing radiation and does not require the use of contrast agents. However, due to the applied magnetic field, MRI requires the use of specific instruments from non-magnetic materials [26], [27] to avoid interference to the images. Therefore, these instruments should be created from MRIcompatible non-magnetic materials.

The design requirement of a tactile sensing device heavily depends on the type of application it will be used for in RMIS. Information about mechanical properties of soft tissue could be used in two ways: either to sense the pressure applied by an instrument, in order not to injure the tissue and blood vessels during manipulation; or to enable the possibility to palpate soft tissues during RMIS to detect sites of abnormalities. In the first case, sensors are mainly used in the design of 
manipulation specific surgical instruments, such as graspers, forceps, cutters and tips for catheters. But in the second case, palpation probes are designed in order to evaluate the mechanical structure of tissue and to detect the presence of abnormalities.

\section{Human TACTILE SENSING}

The studies on tactile technologies were rising along with studies on the human tactile perception [2]. The simultaneous development of these two areas is not a coincidence, as human tactile sensing acts often as a standard for an ideal tactile technology. Therefore, to create an artificial tactile sensing device, it is beneficial to take into account the biological background of tactile sensing, such as the distribution and functionality of mechanoreceptors. These sensing elements are spatially distributed in the soft tissue between the skin, the finger bone and the nail in a strategic way, possibly to take advantage of different tissue dynamics under different probing behaviors.

\section{A. Biological Background of Tactile Sensing}

Humans possess several types of mechanoreceptors ([28], [29]), which respond to different types of mechanical stimuli (Fig. 1). The receptors densely located in the top layer of the skin, Merkel cells, respond to light touch and static stimuli. These cells have a small receptive area and a high sensitivity to small vibrations (less than $5 \mathrm{~Hz}$ ) [30], fine spatial discontinuities (about $0.5 \mathrm{~mm}$ [31]), edges, and the orientation of the examined surface [32], [33]. Ruffini corpuscles are located deeper in the dermis and respond to stretching in the joining tissue, and mostly react to sustained pressure and are used in kinaesthetic sense. Meissner's corpuscles respond to vibrations from 5 to $50 \mathrm{~Hz}$ [30] and are located close to the skin surface. The density of these mechanoreceptors is the highest with the receptors spaced 3 - 5 mm apart. Moreover, Meissner's corpuscles are responsible for slip detection between an object and the skin [34]. The receptors located most deeply (Pacinian corpuscles) respond to high-frequency vibrations and high pressure [35], [36]. These receptors quickly adapt to the stimulus and do not respond to static exposure. The density of these receptors is not very high and the size of the cell is relatively large. According to [37] the average number of Pacinian corpuscles in human hands is 300 cells. In addition, Pacinain corpuscles are producing a response only in case the tactile stimulus is acting on the array of these cells that is on the wider area of the skin [38]. Therefore, human tactile system perceives separate stimuli, such as different frequencies of vibrations, light and moderate touch and deep pressure, with the help of mechanoreceptors, strategically located in the finger. Thus, the tactile perception of an object is formed from the combination of perceived information. More information on human anatomy can be found in [39].

\section{B. Manual Palpation Techniques}

Various strategies taken by expert surgeon in soft tissue manual palpation is another important area that should be taken into account during the development of a medical tactile device for palpation. It can provide guidelines not only for the design of the sensor but also for the optimal control strategies of the examination, which can lead to higher detection rate of tumors [40]. Soft tissue palpation is a mechanically complex process, requiring expertise and continuous training. It is broadly applied for initial breast and prostate examinations to find the presence, location, shape, mechanical properties, and texture of abnormalities. There are several palpation techniques in use, depending on the organ examined and the shape and depth of the abnormality [41], [42]. Palpation methods can be subdivided into three main techniques, such as global movement, local movement and palpation pressure, though they are usually combined in order to achieve the best result [43].

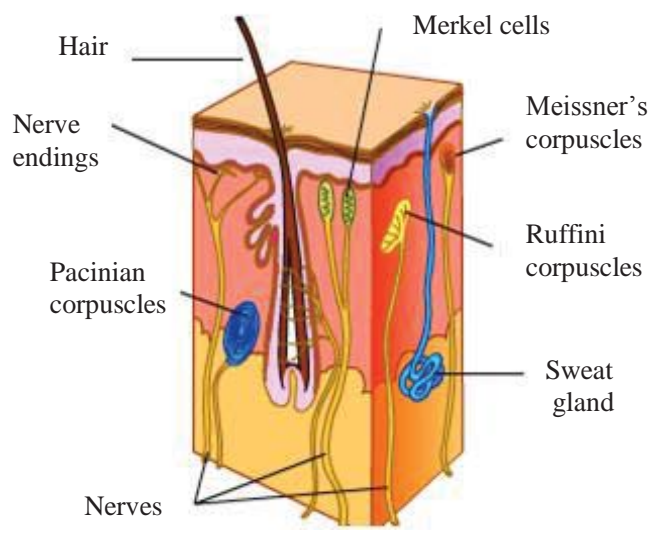

Fig. 1. Cross section of human skin. Picture adapted from [44].

\section{1) Global Movement}

The first technique of manual palpation - global finger movement (GFM) - is aimed to increase palpation efficiency and accuracy by covering the whole surface of the organ. It reduces the risk of missing a suspicious formation. In prostate examinations, this technique is performed with three main different patterns: U, L, V - shape, as well as straight line movement [43]. Clinical breast examinations are usually performed with the following three patterns: concentric circles, radial spokes, and vertical stripes [45]. The pattern choice mainly depends on the preference and training of the examiner.

\section{2) Local Movement}

Based on GFM, the examiner defines the areas of possible abnormalities. For further examination it is necessary to explore those more thoroughly. Therefore, the local finger movement (LFM) method is applied, and performed only within a selected section. This type of palpation helps physicians to understand the shape and depth of an abnormality. Due to different shape and location of organs, the approaches of LFM are specific to the target organ, though they are similar. In particular, three methods of LFM can be outlined for palpation examinations performed with one finger [45]. The first method is tapping - a fast striking discontinuous touching of the tissue. The second technique is vibration, where the finger is kept at constant contact with the tissue and 
the force direction of the finger is varied during examination. Finally, there is the sliding pattern - a smooth movement over a defined area with relatively constant pressure.

\section{3) Palpation Pressure}

The third palpation technique, apart from GFM and LFM, is finger movement pressure (FMP), which corresponds to the average intentional finger pressure applied during the palpation procedure, such as light and deep palpation [46] Light pressure is mainly used with GFM to access the general mechanical properties and temperature of the organs. Indentation of this type of palpation does not exceed $2 \mathrm{~cm}$ and pressure is as light as possible. Deep palpation is performed with heavier pressure, mainly used for LFM, with an indentation of about $4 \mathrm{~cm}$, and is used to evaluate the stiffness, size, contours and shape of the formation or of the organ.

The above information not only represents the complexity of the task to replicate the human-like touch perception mechanism, but also underlines the importance of embodied perception of the environment during tactile examination. This means, that tactile sensing systems should be able to interact with the given environment and use the perceived information about it to change the behavioral pattern of measurement process [47]. This issue becomes more challenging during invivo examination of organs, which represent variable and nonlinear environment.

We believe that it is important to consider the biological background of tactile sensing, such as human tactile perception and the methods of manual palpation in order to develop artificial tactile sensors for RMIS. The measurement of soft tissue properties during manual palpation is an essential step for surgeons. This information should be always taken into account while developing a tactile device for medical applications [48]. Firstly, it can be a valuable source of knowledge for the creation of biologically inspired tactile devices. In addition, the understanding of human tactile sensing can provide researchers with understanding on the design, guidance and control of the tactile device.

TABLE I

WAYS OF CLASSIFICATION OF TACTILE SENSORS FOR SURGICAL APPLICATIONS

\begin{tabular}{ll}
\hline \multicolumn{1}{c}{ Construction principle } & \multicolumn{1}{c}{ Application type } \\
\hline Indentation devices & $\begin{array}{l}\text { Tactile probes for artificial } \\
\text { palpation }\end{array}$ \\
\hline Aspiration or Absorption devices & Tactile probes \\
\hline Catheter tip with sensing element & Catheters \\
\hline Layered structure & Graspers \\
& Forceps \\
\hline Arrayed structure & Cutters \\
\hline Non-contact method & Tactile probes \\
\hline Combination of several design & Various applications \\
principles & Tactile probes \\
\hline \hline
\end{tabular}

\section{Design of TACtile And Force Sensing DeVICES}

In this section the structural design and sensing principles of medical tactile sensors for RMIS is presented. We review the tactile devices, developed for use in RMIS and other related surgical applications, during the recent decade, which the authors believe are the most significant works that are acting towards the development of the field.

Considering the fact that tactile devices are directly interacting with soft tissue, their design is one of the factors that can influence the whole results of examination. The following sections provide an overview of the tactile sensors based on their construction principle and application type for surgery. In addition the sensing principles and the associated features of each design will be discussed.

Table I classifies tactile sensing devices designed for surgical applications. The examples of the devices will be presented hereafter.

\section{A. Contact Devices, Based on the Indentation Principle}

1) Common Design Principles of Indentation Devices

The most popular design of tactile devices in surgical applications is the contact probe, which is akin to the human finger, measuring indentation during probing. Such construction principle is usually employed for probing devices, which directly serve the purpose of artificial tactile probing or palpation. The typical application of such a device is the detection and localization of tissue abnormalities, such as malignant or benign formation. A certain amount of force is applied on the target tissue, which results in changes of stress and strain values. Then a finger-like indentation sensing device measures the stiffness of a soft tissue.

The shape of the indenter, which is the main construction element, plays an important role in the results of examination. Theoretically, to get the most accurate measurements from inhomogeneous material, the contact area of measurement should be minimal. However, the shape of an indenter should be designed in such way, that it does not damage tissue during the surgery, this means, its dimensions should be appropriate for the target tissue not to be torn or damaged. Moreover, for a complete organ representation, the measurement should be performed continuously over the surface of an organ. In addition this shape is similar to human fingertip, which is actively used to detect tactile information during manual palpation. The diameter of the sphere should be chosen according to the viscoelastic properties of the tissue - very large diameter requires higher loads and more effort during measurements, while too small diameter will not provide desired dynamical properties for the measurement. It has been proven, that depth and stress of indentation is inversely related to the diameter of the indenter, but this relationship is nonlinear [49].

\section{2) Transduction Principles}

The tactile devices, based on the indentation construction principle allow using different transduction principles, which are discussed below. Force feedback measurement is one of the most wide-spread methods for soft tissue mechanical property examination, as it is simple, accurate and convenient for the use during RMIS.

Commercially available sensors offer easy and accurate force data measuring, but they can be unsuitable size, made 
from MRI incompatible material. The most popular commercial device, used for indentation device is threedimensional force and torque sensor NANO 17 (ATI industrial automation), which provides good accuracy and sensitivity along with miniature size (17 $\mathrm{mm}$ in diameter). The examples of indentation devices using this sensor can be found in [50], [51].

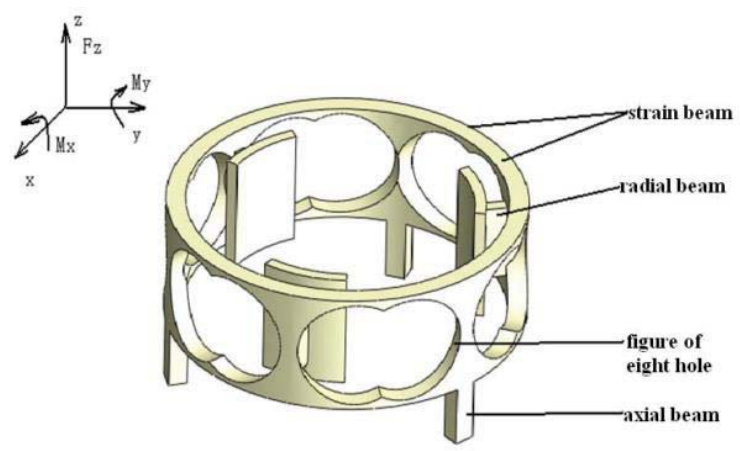

Fig. 2. Design principle of sensing structure in [52].

To reduce the cost, as well as to combine the accuracy and miniature size for the application in RMIS, there is a need for the development of tactile devices using specially developed transducers. For instance, one can use strain gauges to measure force feedback. The advantage of such method is relative flexibility of the construction and therefore the possibility to design multi - dimensional sensors. An example of an indentation probe, based on strain gauges can be found in [52] (Fig. 2), where a three-dimensional force and torque sensor was developed for arthroscopic surgery. The specific feature of this design is hollow structure of the sensor, which allows to place the sensor on the tip of the probe and to enable use of visual feedback necessary for this type of surgery. Strain gauges are relatively cheap, and this allows disposing the probe after use. However, such sensors cannot be used inside MRI scanner and are sensitive to temperature changes. The same limitations can be applicable also for the sensors using piezoelectric effect [53], [54].

Among the works devoted to tactile sensors, one can outline the indentation devices that work using fiber optical principle, where the modulation of light intensity is used to represent the variable force feedback from the soft tissue [55]-[57]. They are compatible with MRI, as the transducer is made of nonmagnetic materials. In addition, fiber optical transducers can work with no wiring and are resistive to temperature variations. The construction principle of the fiber-optical force sensor requires converting force feedback into displacement that is usually achieved by the means of deforming an elastic part of the sensor. The choice of elastic material becomes a very important issue in the sensor construction, as the properties of the material will define sensor accuracy parameters. Nonlinear behavior and response time of elastic part are the important factors that should be considered during sensor calibration. The deformation of elastic tip of the sensor can also be used to measure tactile data using optical information. For instance, work in [58] describes the compliant optical tactile sensor, where the shape change of transparent elastomer is measured by high speed image sensor.

Alternative, but expensive, sensing principle is based on micro-fiber Bragg grating (FBG), which gives better accuracy of estimating stiffness parameters in addition to being a miniature solution. The specific feature of FBG is variation of the refractive index, and thus the length of the wavelength, on the small section of the fiber. An example of such sensor can be found in [59], where the designed sensor, $2.4 \times 2.4 \mathrm{~mm}^{2}$ in size, shows good balance between sensitivity range and linearity. In this example FBG was embedded in the flexible silicone material and was found suitable for the force measurement in RMIS.

As the current state of arts shows, fiber-optical transduction principle allows building miniature multi-modal sensors. The example of three-axis sensor can be found in [60] and six-axis in [61], where the size of the sensor is brought up to $11 \times 10 \times$ $6 \mathrm{~mm}^{3}$ with the help of linear polarizers. However, if these sensors are employed for indentation devices, the performance and accuracy will be largely dependent on the design parameters of the probe. In addition, the structure of fiberoptical transducer can be relatively fragile and may be damaged due to excessive loads.

The indentation devices, which are measuring only force feedback from the target material, are suitable for static onepoint measurements. Most of force feedback-based sensors are measuring just tissue response force and the indentation depth during the measurement is assumed to be constant. This assumption leads to inaccuracy in the estimation of tissue mechanical properties. In order to estimate correct soft tissue parameters, the device should be able to measure both force feedback and indentation depth simultaneously. Alternatively, the indentation depth can be kept constant during measurement, but this approach can be hardly implemented for real surgical applications. The work described in [62] (Fig. 3 ) is based on the optical fiber force sensing, but its construction principle does not use deformable elastic material for the modulation of intensity. Instead, a spherical indenter of the probe is pressed down to the target tissue by air flow and in non-contact position sphere is located on the fixed distance from the optical fiber. During measurements, variation in the tissue stiffness is causing change in the distance between the sphere and the fiber. Thus tissue stiffness is obtained by detecting indentation depth and force feedback. However, the relationship of airflow and non-linear soft tissue responses should be taken into consideration during sensor calibration. Another example of sensor detecting two measurement variables simultaneously can be found in [63]. The third example of stiffness sensor (Fig. 4), which may be in the interest of the reader, is based on the induction principle, where direction and amount of motion is measured along with force feedback [64]. This method is not MRI compatible and is not very popular for the indentation devices, but the results show its good accuracy parameters. 

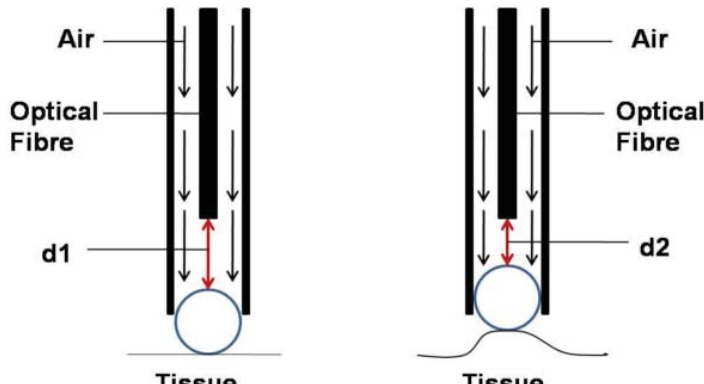

Fig. 3. Sensing principle of stiffness sensor measuring based on optical fiber and air flow [62].

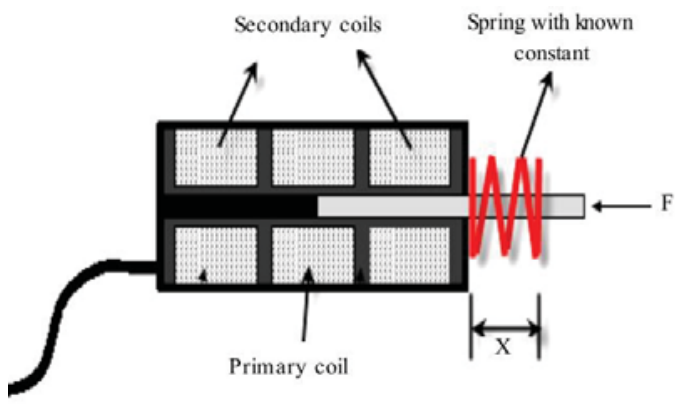

Fig. 4. Measurement principle of tactile indenter, which is based on the induction principle [64].

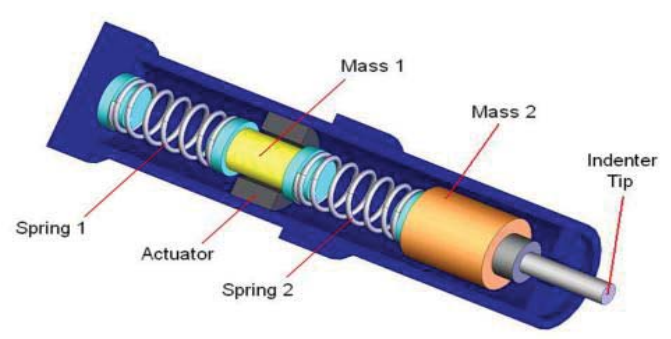

Fig. 5. Design principle of an indentation device which is measuring soft tissue data based on resonance-frequency method [66].

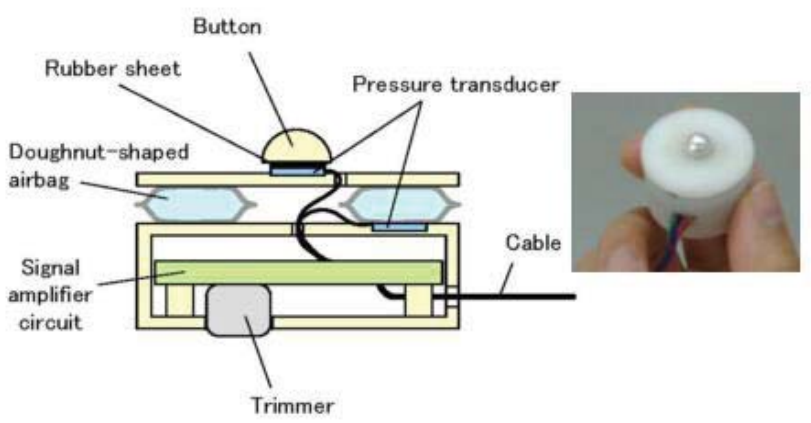

Fig. 6. Schematic design and a prototype indentation device to measure liver stiffness [68].

Apart from force sensing method, resonance-frequency based method can be used. However, at the moment one cannot outline an indentation device, which is capable of measuring mechanical properties of soft tissue over continuous path. For example, work described in [65] uses commercial resonance sensor Venutron ${ }^{\circledR}$, where the static measurements of soft tissue phantoms are performed during controlled indentation depth. The indentation devices based on the combination of mass-spring model with LVDT (low voltage differential transducer) coils [66], [67], sample design principle is shown in Fig. 5. In that case the system is measuring a shift of resonance frequency during indentation process. The main issues in the application of such devices are the measurement repeatability, reliability and dependence on the orientation of the probe. This may lead to occurrence of inaccuracies during in-vivo application of the tactile device. However, the significant advantage of resonant method is the capability to measure both elastic and viscose properties of soft tissues.

In addition to the above presented transduction principles, one can outline a set of papers about indentation devices, which are using application-specific design. The characteristic feature of such devices is the limitation of its implementation method and design constraints, as a physical principle of construction does not allow any modifications. An example of such indentation device can be found in [68], (Fig. 6), it is using ring shaped airbag to detect contact force by measuring variable air pressure and to measure stiffness of liver. The use of air bag allows measuring force feedback in arbitrary direction and to minimize the effect of variable indention.

In [69] the concept of wireless palpation was introduced. Magnetically actuated wireless probe measures stiffness using indentation in a vertical direction. The device does not require a separate trocar port, but is influenced by vibrations and uncertainties of the environment that can affect the accuracy.

To sum up the information about indentation devices used for soft tissue probing or palpation, one can see the active development of them along with requirement for better devices, which would ensure accurate measurements over the whole surface of an organ in real-time.

\section{B. Aspiration Devices}

Another design method, which can be used for tactile examination of soft tissue, particularly stiffness estimation, is the aspiration or suction of target tissue with a pipette device. Such approach is used to perform biopsy for tumor and infection diagnosis [70]. This method is not very well represented in the scientific literature for the purpose of soft tissue palpation. Although, there are several advantages: aspiration devices are MRI compatible, the pipette can be sterilized, and, importantly, it is able to estimate the value of Young's modulus instead of the relative stiffness parameter of the area. There are no examples of such tactile devices developed directly for RMIS, but one can mention several interesting works describing this principle on soft tissue examination [71]-[73].

In order to implement the measurement of stiffness the device should be in direct contact with the tissue, as the air from the pipette is sucked out. Then, there should be a system to measure the aspirated level of soft tissue, assumed to be homogeneous, which is then analyzed to estimate Young's modulus (Fig. 7). As a downside of this method one can mention the constraint to perform fast measurements over an organ area, due to necessity to aspirate and then to release the target area. In addition, to create a full representation of an 
organ, or so-called stiffness distribution map, the positioning of pipette should be very accurate in order to gather the information from the whole organ. Finally, the design of a pipette device is directly influencing the accuracy of the measurements. The diameter of an aspirated area and wall thicknesses of a pipette are important parameters. More information about design can be found in [71].

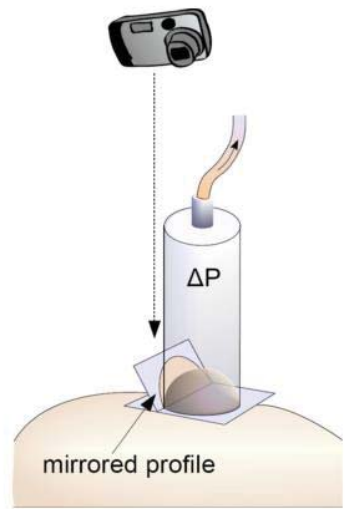

Fig. 7. Measurement principle of an aspiration device: $\Delta P$ - aspiration pressure, mirrored profile of aspirated tissue area is captured by camera [72].

\section{Catheters with Tactile Sensing Elements}

Catheters are broadly used for applications in minimally invasive cardiac surgeries to perform various manipulations, including coronary artery bypass surgery, procedures on beating heart, and mitral valve surgeries [74]. During catheterization procedures, it might be desired to control the applied force during catheter insertion to avoid damage of the vessels or chambers as well as to distinguish between the stiffness of soft tissues by the employing the tip of catheter with force sensor. Here it is worth to mention, that in case of catheterization procedures the tactile device is mostly used in order to evaluate the strength of applied force or to detect the presence of an occurred contact, and scanning of soft tissue properties is not needed.

In addition, catheterization procedures often require use of MRI during surgery; therefore, tactile sensors employed in catheters should not contain any metal parts. The analysis of the devices, using fiber optical sensors shows, that this type of sensing is feasible for different applications in RMIS, including catheterization procedures. The example of the catheter with such transduction principle can be found in [75] and in [76]. Sensor described in [77] uses expansion of the soft and flexible balloon with fluid for tactile information sensing. The pressure and flow of fluid is changing depending on the load applied and measured by flow and pressure sensors outside the setup. However, the difficulty of such setup is in the maintaining constant flow rate in the sensor and overcoming problem of the occurring slip.

As practice shows, the most successful devices can be developed employing combined sensing and mechanical principles. For example, in [78] as shown on Fig. 8, a hybrid sensor developed for catheterization procedures uses both the piezoresistive effect and optical fiber sensing. The sensor is capable of detecting the deformation of an object via the deformation of reflective diaphragm by the means of optical force sensing, as well as force feedback using the piezoresistive film. Thus, the relative stiffness of contact object can be obtained.

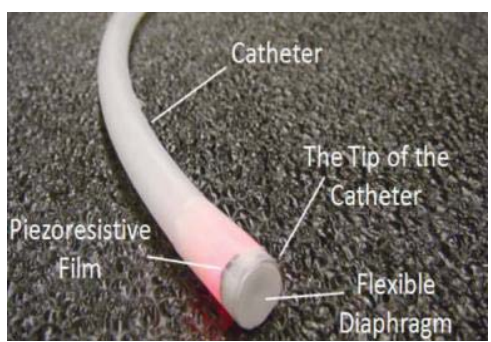

Fig. 8. Catheter tip, which is measuring relative stiffness of contact tissue [78].

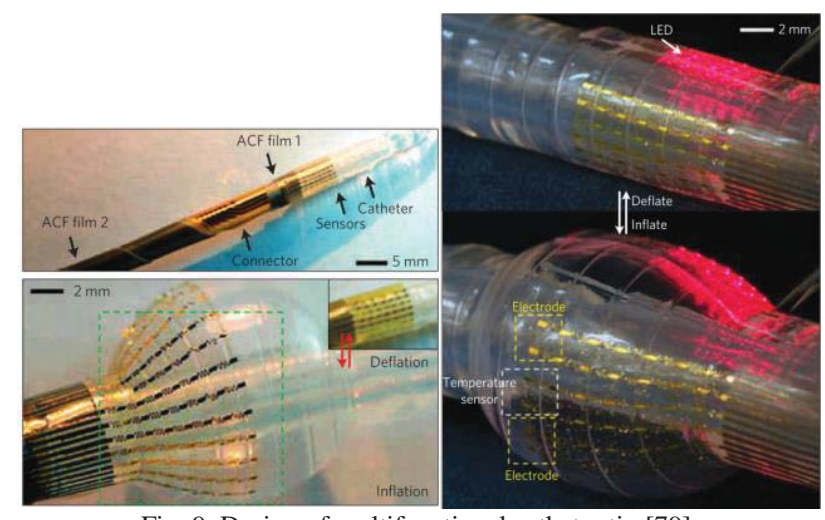

Fig. 9. Design of multifunctional catheter tip [79].

In the context of tactile devices developed for catheters it is necessary to mention the work described in [79], where multifunctional balloon catheter is presented - Fig. 9. Original method of fabrication allowed employing tactile, temperature and flow sensors. In addition, the device showed good accuracy parameters and was tested in-vivo that is a necessary step towards the implementation of the device for the real surgery.

\section{Layered Structure}

This section discusses another design approach for tactile sensors design - layered structures, which represents a device, composed of various materials or transducers placed one upon another. The method has got various advantages, as several transduction principles as well as different materials can be combined. Tactile sensors built on the layer principle can be successfully integrated in a surgical instrument, such as graspers, as well as for tactile probes used for soft tissue exploration.

Tactile sensing devices employing various materials in a layered configuration can be compared with a structure of human finger, which is composed of different layers, possessing various mechanical properties and acting differently during object exploration [80]. Similarly, tactile sensors with layered structure can use a layer of silicone or rubber material. However, it is necessary to take into account the viscoelastic properties of measured soft tissue during choice of soft material for the sensor layers, because the sensitivity of the device will depend on the mechanical interaction between the tool and tissue. For instance, in [81], 
[82], the choice of soft material is directly influencing the characteristics of tactile device, as the elastic part of the device is in direct contact with measured material.

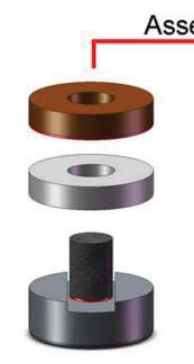

Exploded view

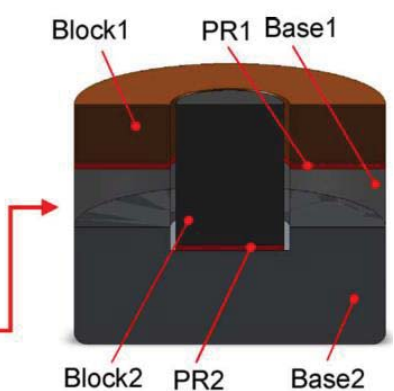

Block2 PR2 Base2

Fig. 10. Layered structure of soft tissue sensor: Block 1 and Block 2 are composed from rubber with different Young's modulus, PR2 piezorezistive film, Base1 and Base2 - steel components [82].

The combination of various materials in one device, allows measuring not just feedback force, but also the stiffness of the tissue. In [82], Fig. 10, two rubber blocks with different values of Young's modulus are placed concentrically upon pressure sensing elements, that allow to determine the Young's modulus of soft tissue. Another example is micro electromechanical systems (MEMS) stiffness sensor in layered structure, which measures soft tissue properties based on a relative displacement of two elements with the help of capacitive sensing membrane [83]. MEMS technologies have a good potential for various sensing applications, however the current fabrication process is relatively difficult [84].

The viscoelastic properties of the outer surface of a tactile sensing device could complicate the possibility to perform fast movements over the organ due to friction. Thus, such structure is more suitable for static point measurements of soft tissue and can be successfully integrated in surgical instruments. Surgical graspers equipped with tactile feedback are able to provide surgeon with information about grasping force as well as to detect the presence of small blood vessels, thus reducing the risk of damaging soft tissue. Such devices with sensing capabilities can estimate tactile information more accurately, as the target area is fixed between the instrument and no significant displacement occurs. As it is seen from relevant examples of graspers [85], [86], the elastic material incorporated in a layer can enhance stability of a grasp, and can even form teeth of an instrument. However, variability of data can be induced from vibration or shaking of an instrument.

The typical transducer used for a design of a sensor composed of different layers should be thin and robust, for example it can be piezoresistive films [81], [82], [85], PVDF (polyvinylidene fluoride) films [86], [87], capacitive sensing elements [78] or use of conductive polymer with electrodes [88]. However, all these transducers do not enable MRI compatibility, which is sometimes necessary for the use during RMIS. In [89] the tactile sensor uses fiber optical sensing principle (Fig. 11). The specific characteristic of this layered sensor is composed of three fibers sandwiched between perpendicular rods of different lengths and several elastic materials. The configuration of rods allows measuring the relative deflection of the sensor structure, therefore detecting the tactile information from the contact surface.

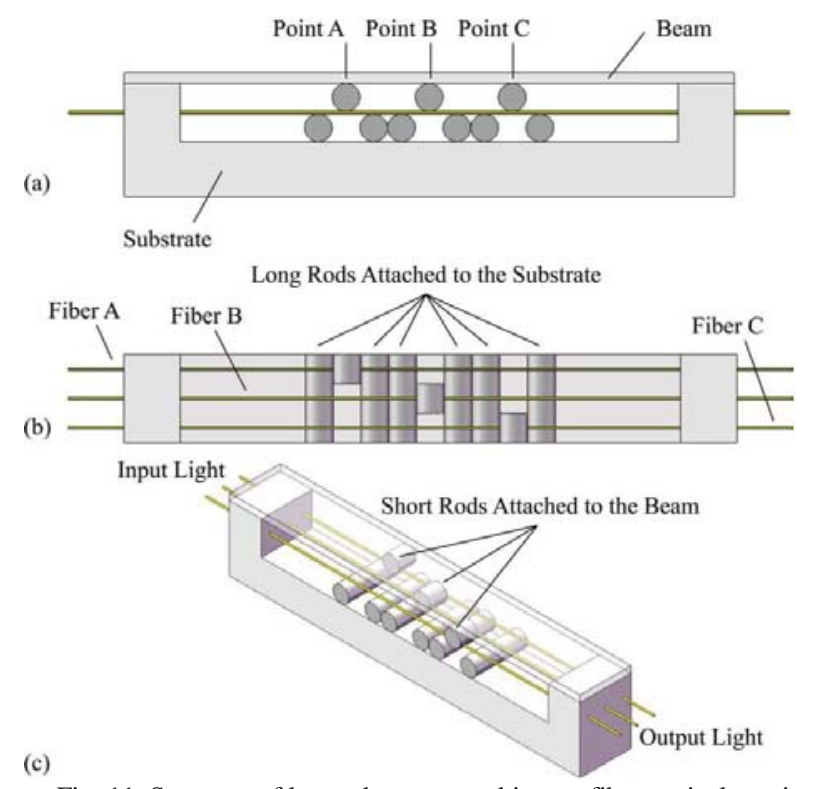

Fig. 11. Structure of layered sensor working on fiber-optical sensing principle [89].

Layered structure of a tactile sensor allows measuring the stiffness of a soft tissue without the information about indentation depth. However, the fabrication process of layers can be relatively complex. In addition, such design is more suitable for stable static one-point measurements, where it is able to provide accurate results. Therefore, one can conclude, that the best application of a layered tactile device is integration of it in a surgical instrument, such as graspers or forceps.

\section{E. Array Sensors}

Tactile devices, designed as spatially distributed sensing elements in arrays, can be very advantageous for RMIS. Such design of tactile principle can be compared with the perception of a fingertip, which is using multiple mechanoreceptors. The structure of sensor array can be built up from different transducers and their combination, as well as various tactile sensors can be united in one sensing array. One of the advantages of arrayed tactile sensor is the possibility to use its output directly as the visual information [88] or to the tactile display [90]-[92].

The sensitivity of the array depends on the number of sensing elements. However, the increase in their number can lead to the bigger size and weight of the device, which is undesirable in case of surgical instruments. Therefore, one needs to use small sized transducers to increase the sensitivity of the device. For example, in [93] this problem was solved using an array of conductive microchannels embedded in an elastic deformable environment. Because of such technology, the sensor is suitable to be used on surgical microgrippers (typical width of an instrument is $1 \mathrm{~mm}$ ). Another perspective technology is the use of MEMS [94], [95], which has got various advantages such as easy production and miniature size, and can be easily integrated in a surgical instrument. The 
most suitable transducers for this approach can be PVDF films [94] and capacitors [96] due to their small size. In [95] the measurement sensitivity of MEMS stiffness sensor has been improved employing an array of force sensing units with two different mechanical stiffness parameters. Thus, a sensing diaphragm was created and can be integrated in a surgical grasper.

In addition, the arrayed structure of a tactile sensor allows the flexibility of the device and, therefore, the possibility of it to adapt to the environment. Good examples, proving the feasibility of this approach can be found in the works about the development of robotic skin [97]-[100], where authors present the tactile sensing structure that is able to cover large curved areas. Forward-looking, this approach can be integrated for the use in RMIS, as flexible structure can be used to cover the surface of an organ. However, miniaturization of this approach requires further research.

\section{F. Non-contact Devices}

As an alternative to contact methods, stiffness can also be measured using noncontact devices. This method can be used only for devices that are developed for the localization and detection of hard tissue areas, and it is not applicable for integration in the instruments. The basic working principle of this methodology is based on the detection and measurement of applied deformation and resulting indentation. The advantage of this method is the possibility to measure properties of soft tissue over continuous path in real-time, in addition, the effect of mechanical friction from tool and tissue interaction cannot influence the dynamics of the movement.

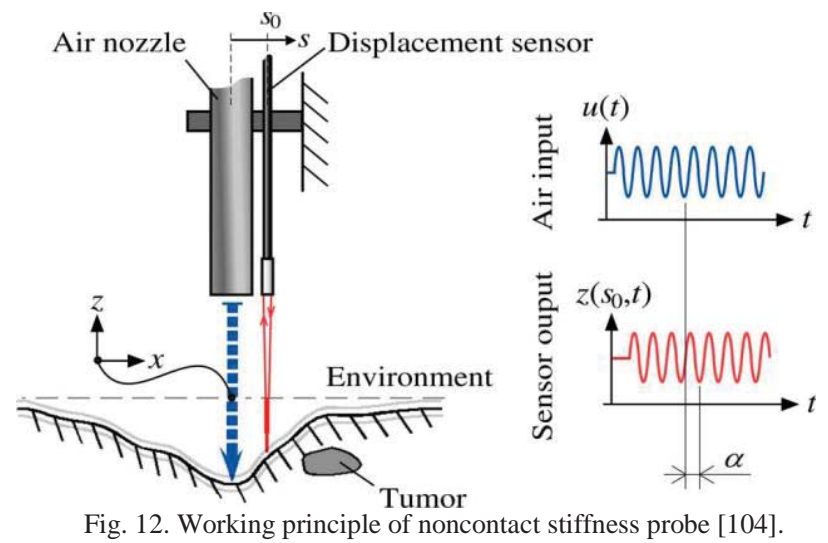

The most convenient approach to indent the tissue in noncontact way is the application of an air jet to the target tissue [101], [102]. This can be easily achieved in the operation theatre, as air supply is broadly implemented during the surgery. The pressure of an air jet can be regulated, thus it is possible to control the flow applied to the tissue and to reduce the risk of tissue damage during examination. The effect of resulting indentation can be estimated using visual feedback system. In [103] similar approach is applied to solve the inverse problem of estimation of the viscoelastic parameters. However, the main limitation of vision based indentation evaluation is the necessity to keep camera position fixed with respect to organ surface and to avoid shaking and vibration of the device. This is not possible during the real surgery due to the natural movement of internal organs. In addition, the data processing during this type of measuring is time-consuming.

Another way of noncontact measurement of soft tissue parameters was proposed in [104], where a fiber optical displacement sensor is used to estimate the changes in the tissue, which is assumed to be homogeneous. In this work (Fig. 12) in order to detect tumor, phase shift difference between air pressure input and displacement sensor output are measured. Therefore, the change in stiffness between two measurements will be detected.

\section{CONCLUSIONS}

From the information presented in this paper, we can conclude, that at the moment there exist relatively robust devices, which are capable to provide accurate tactile information during static measurements from one point. However, as it was already mentioned in the paper, to detect the information about mechanical parameters of an organ, it is required to scan soft tissue in a dynamic way. Therefore, it is beneficial to develop devices and algorithms, which could operate in real-time and provide stable measurements. Thus, a device should be able to adapt to the variable conditions of the environment [105], [106]. This includes the accounting on the force applied, soft tissue restitution dynamics versus time, the shape of an organ and many other factors. In addition, as it was shown, the type of application of tactile device is an important factor, which is the main factor defining design of the tool.

One can observe, at the present moment there exist only few devices approved during clinical in-vivo studies. This can be justified by the fact, that only robust and safe devices, which fulfill all surgical requirements, can be tested on live organism. These tests are very important, as the properties of tissue in living conditions differ from the properties of ex-vivo tissues. This is the last and most significant stage of the development of a tactile device, and it requires preparation and meticulous work.

\section{ACKNOWLEDGMENT}

We thank the medical and engineering community for their useful comments. The work described in this paper is partially funded by the Seventh Framework Programme of the European Commission under grant agreement 287728 in the framework of EU project STIFF-FLOP, and by the National Institute for Health Research Biomedical Research (NIHR) Centre based at Guy's and St Thomas' NHS Foundation Trust and King's College London. The views expressed are those of the authors and not necessarily those of the NHS, the NIHR or the Department of Health. We also acknowledge the Guy's and St. Thomas' Charity, Vattikuti Foundation, MRC Centre for Transplantation. 


\section{REFERENCES}

[1] Y.-J. Shiah, F. Chang, and W.-C. Carl Tam, "Recognition of tactile relief by children and adult," Percept. Mot. Skills, vol. 113, no. 3, pp. 727-38, 2011.

[2] M. H. Lee and H. R. Nicholss, "Review Article Tactile Sensing for Mechatronics - a State of the Art Survey," Mechatronics, vol. 9, no. 1, pp. 1-31, Feb. 1999.

[3] P. Puangmali, K. Althoefer, L. D. Seneviratne, D. Murphy, and P. Dasgupta, "State-of-the-Art in Force and Tactile Sensing for Minimally Invasive Surgery," IEEE Sens. J., vol. 8, no. 4, pp. 371381, Apr. 2008.

[4] S. Schostek, M. O. Schurr, and G. F. Buess, "Review on Aspects of Artificial Tactile Feedback in Laparoscopic Surgery," Med. Eng. Phys., vol. 31, no. 8, pp. 887-98, Oct. 2009.

[5] M. Eltaib, "Tactile sensing technology for minimal access surgery-a review," Mechatronics, vol. 13, no. 10, pp. 1163-1177, Dec. 2003.

[6] J. Dargahi and S. Najarian, "Human tactile perception as a standard for artificial tactile sensing - a review," Int. J. Med. Robot. Comput. Assist. Surg., vol. 01, no. 01, pp. 23-35, 2004.

[7] R. S. Dahiya, G. Metta, M. Valle, and G. Sandini, "Tactile SensingFrom Humans to Humanoids," IEEE Trans. Robot., vol. 26, no. 1, pp. 1-20, Feb. 2010.

[8] H. Yousef, M. Boukallel, and K. Althoefer, "Tactile sensing for dexterous in-hand manipulation in robotics - A review," Sensors Actuators A Phys., vol. 167, no. 2, pp. 171-187, Jun. 2011.

[9] D. B. Camarillo, T. M. Krummel, and J. K. Salisbury, "Robotic technology in surgery: past, present, and future.," Am. J. Surg., vol. 188, no. 4A Suppl, p. 2S-15S, Oct. 2004.

[10] B. M. A. Schout, A. J. M. Hendrikx, F. Scheele, B. L. H. Bemelmans, and A. J. J. A. Scherpbier, "Validation and implementation of surgical simulators: a critical review of present, past, and future.," Surg. Endosc., vol. 24, no. 3, pp. 536-546, 2010.

[11] H. G. Kenngott, L. Fischer, F. Nickel, J. Rom, J. Rassweiler, and B. P. Müller-Stich, "Status of robotic assistance-a less traumatic and more accurate minimally invasive surgery?," Langenbecks. Arch. Surg., vol. 397, no. 3, pp. 333-41, Mar. 2012.

[12] G. Perigli, C. Cortesini, E. Qirici, D. Boni, and F. Cianchi, "Clinical benefits of minimally invasive techniques in thyroid surgery.," World J. Surg., vol. 32, no. 1, pp. 45-50, Jan. 2008.

[13] C. E. Reiley, T. Akinbiyi, D. Burschka, D. C. Chang, A. M. Okamura, and D. D. Yuh, "Effects of Visual Force Feedback on Robot-assisted Surgical Task Performance,” J. Thorac. Cardiovasc. Surg., vol. 135, no. 1, pp. 196-202, Jan. 2008.

[14] B.-M. Ahn, J. Kim, L. Ian, K.-H. Rha, and H.-J. Kim, "Mechanical property characterization of prostate cancer using a minimally motorized indenter in an ex vivo indentation experiment.," Urology, vol. 76, no. 4, pp. 1007-11, Oct. 2010.

[15] J. I. Lopez, I. Kang, W.-K. You, D. M. McDonald, and V. M. Weaver, "Integrative Biology In situ force mapping of mammary gland transformation," Society, pp. 910-921, 2011.

[16] G. Tholey, J. P. Desai, and A. E. Castellanos, "Force Feedback Plays a Significant Role in Minimally,” Ann. Surg., vol. 241, no. 1, pp. $102-$ 109, 2005.

[17] C. King, M. O. Culjat, M. L. Franco, C. E. Lewis, E. P. Dutson, W. S. Grundfest, and J. W. Bisley, "Tactile Feedback Induces Reduced Grasping Force in Robot-Assisted Surgery," in IEEE transactions on haptics, 2009, vol. 2, no. 2, pp. 103-110.

[18] F. G. Duhaylongsod, "Minimally invasive cardiac surgery defined.," Arch. Surg., vol. 135, no. 3, pp. 296-301, Mar. 2000.

[19] D. Mckay and G. Blake, "Optimum incision length for port insertion in laparoscopic surgery," Ann. R. Coll. Surg. Engl., vol. 88, no. 1, p. 78, 2007.

[20] M. M. Bhandari A, Hemal A, "Instrumentation, sterilization, and preparation of robot,” Indian J. Urol., vol. 21, pp. 83-88, 2005.

[21] R. A. Kolstad, "How well does the Chemiclave sterilize handpieces?," J. Am. Dent. Assoc., vol. 129, no. 7, pp. 985-91, Jul. 1998.

[22] J. E. Sebben and M. D. Davis, "Sterilization and care of surgical instruments and supplies," J. Am. Acad. Dermatology, vol. 11, no. 3, pp. 381-392, 1984

[23] R. J. Lederman, "NIH Public Access," Circulation, vol. 112, no. 19, pp. 3009-3017, 2005.

[24] A. E. Sloan, M. S. Ahluwalia, J. Valerio-Pascua, S. Manjila, M. G. Torchia, S. E. Jones, J. L. Sunshine, M. Phillips, M. a Griswold, M. Clampitt, C. Brewer, J. Jochum, M. V McGraw, D. Diorio, G. Ditz, and G. H. Barnett, "Results of the NeuroBlate System first-in-humans Phase I clinical trial for recurrent glioblastoma," J. Neurosurg., vol. 118, no. 6, pp. 1202-19, Jun. 2013.

[25] E. Hempel, H. Fischer, L. Gumb, T. Hohn, H. Krause, U. Voges, H. Breitwieser, B. Gutmann, and J. Durke, "An MRI-Compatible Surgical Robot for Precise Radiological Interventions," Comput. Aided Surg., vol. 8, pp. 180-191, 2003.

[26] V. Seifert, M. Zimmermann, C. Trantakis, H. E. Vitzthum, K. Kühnel, a Raabe, F. Bootz, J. P. Schneider, F. Schmidt, and J. Dietrich, "Open MRI-guided neurosurgery.," Acta Neurochir. (Wien)., vol. 141, no. 5, pp. 455-64, Jan. 1999.

[27] N. Yu, C. Hollnagel, A. Blickenstorfer, S. S. Kollias, and R. Riener, "Comparison of MRI-Compatible Mechatronic Systems With Hydrodynamic and Pneumatic Actuation," IEEE/ASME Trans. Mechatronics, vol. 13, no. 3, pp. 268-277, Jun. 2008.

[28] A. B. Vallbo and R. S. Johansson, "Properties of cutaneous mechanoreceptors in the human hand related to touch sensation.," Hum. Neurobiol., vol. 3, no. 1, pp. 3-14, Jan. 1984.

[29] R. S. Johansson and A. B. Vallbo, "Tactile sensory coding in the glabrous skin of the human hand," Rends Neurosci., vol. 6, no. 1, pp. 27-32, 1983.

[30] E. B. Goldstein, Sensation \& Perception, vol. 22. WadsworthThomson Learning, 2004, pp. 1-459.

[31] K. O. Johnson, "The roles and functions of cutaneous mechanoreceptors.," Curr. Opin. Neurobiol., vol. 11, no. 4, pp. 45561, Aug. 2001.

[32] J. R. Phillips and K. O. Johnson, “Tactile spatial resolution. II. Neural representation of Bars, edges, and gratings in monkey primary afferents.," J. Neurophysiol., vol. 46, no. 6, pp. 1192-1203, 1981.

[33] R. S. Johansson, U. Landstrom, and R. M. Lundstrom, "Sensitivity to Edges of Mechanoreceptive Afferent Units Innervating the Glabrous Skin of the Human Hand," Brain Res., vol. 244, pp. 27-32, 1982.

[34] M. A. Srinivasan, J. M. Whitehouse, and R. H. LaMotte, "Tactile detection of slip: surface microgeometry and peripheral neural codes.," J. Neurophysiol., vol. 63, no. 6, pp. 1323-1332, 1990.

[35] A. Prevost, J. Scheibert, and G. Debrégeas, "Effect of fingerprints orientation on skin vibrations during tactile exploration of textured surfaces," Commun. Integr. Biol., vol. 2, no. 5, pp. 422-424, 2009.

[36] B. L. Munger and C. Ide, "The enigma of sensitivity in Pacinian corpuscles: a critical review and hypothesis of mechano-electric transduction.," Neurosci. Res., vol. 5, no. 1, pp. 1-15, Oct. 1987.

[37] B. Stark, T. Carlstedt, R. Hallin, and M. Risling, "Distribution of human pacinian corpuscles in the hand A cadaver study," J. Hand Surg. J. Br. Soc. Surg. Hand, vol. 23, no. 3, pp. 370-372, 1998.

[38] F. Vega-Bermudez and K. O. Johnson, "SA1 and RA receptive fields, response variability, and population responses mapped with a probe array.," J. Neurophysiol., vol. 81, no. 6, pp. 2701-2710, 1999.

[39] S. Standring, Gray's Anatomy: The Anatomical Basis of Clinical Practice, vol. 10. 2008, p. 1600 .

[40] J. Konstantinova, M. Li, V. Aminzadeh, P. Dasgupta, K. Althoefer, and T. Nanayakkara, "Force-Velocity Modulation Strategies for Soft Tissue Examination," in IEEE International conference on intelligent Robots and systems, 2013, pp. 1998-2003.

[41] L. Bendtsen, R. Jensen, N. K. Jensen, and J. Olesen, "Pressurecontrolled palpation: a new technique which increases the reliability of manual palpation," Cephalalgia, vol. 15, no. 3, pp. 205-10, Jun. 1995.

[42] L. E. Eberman and M. E. Finn, "Enhancing Clinical Evaluation Skills: Palpation as the Principal Skill," Athl. Train. Educ. J., vol. 5, no. 4, pp. 170-175, 2010.

[43] N. Wang, G. J. Gerling, R. M. Childress, and M. L. Martin, "Quantifying Palpation Techniques in Relation to Performance in a Clinical Prostate Exam," IEEE Trans. Inf. Technol. Biomed. a Publ. IEEE Eng. Med. Biol. Soc., vol. 14, no. 4, pp. 1088-97, Jul. 2010.

[44] M. L. Cain, H. Damman, R. A. Lue, and C. K. Yoon, Discover Biology. W. W. Norton \& Company, 2000, pp. 1-4.

[45] K. J. Saunders, C. A. Pilgrim, and H. S. Pennypacker, "Increased Proficiency of Search in Breast Self-examination," Cancer, vol. 58, no. 11, pp. 2531-7, Dec. 1986.

[46] C. Volpicello, "Illustrated Manual of Nursing Practice," AORN, vol. 54, no. 6, p. 1302, 1991.

[47] N. F. Lepora, U. Martinez-hernandez, S. Member, and T. J. Prescott, "Active touch for robust perception under position uncertainty," in IEEE International conference on Robotics and Automation ICRA, 2013, pp. 3005-3010. 
[48] J. Konstantinova, K. Althoefer, P. Dasgupta, and T. Nanayakkara, "Salient Features of Soft Tissue Examination Velocity during Manual Palpation," in Hamlyn Simposium 2013, 2013, pp. 41-42.

[49] A. R. Karduna, H. R. Halperin, and F. C. Yin, "Experimental and numerical analyses of indentation in finite-sized isotropic and anisotropic rubber-like materials.," Ann. Biomed. Eng., vol. 25, no. 6, pp. 1009-16, 1997.

[50] E. Samur, M. Sedef, C. Basdogan, L. Avtan, and O. Duzgun, “A robotic indenter for minimally invasive characterization of soft tissues," Int. Congr. Ser., vol. 1281, pp. 713-718, May 2005.

[51] H. Liu, D. P. Noonan, B. J. Challacombe, P. Dasgupta, L. D. Seneviratne, and K. Althoefer, "Rolling Mechanical Imaging for Tissue Abnormality Localization during Minimally Invasive Surgery," IEEE Trans. Bio-medical Eng., vol. 57, no. 2, pp. 404-14, Feb. 2010.

[52] Z. Cui, Z. Han, H. Pan, and Y. Shao, "Design of a 3-axial Force / Torque Sensor for Arthroscopy Force Sensing," in International Conference on Mechatronics and Automation, 2011, pp. 243-248.

[53] H. Yegingil, W. Y. Shih, and W.-H. Shih, "Probing model tumor interfacial properties using piezoelectric cantilevers.," Rev. Sci. Instrum., vol. 81, no. 9, pp. 095104-9, Sep. 2010.

[54] D. O. Uribe, R. Stroop, and J. Wallaschek, "Piezoelectric self-sensing system for tactile intraoperative brain tumor delineation in neurosurgery.," in Conference of the IEEE Engineering in Medicine and Biology Society., 2009, vol. 2009, pp. 737-40.

[55] P. Puangmali, H. Liu, L. D. Seneviratne, P. Dasgupta, and K. Althoefer, "Miniature 3-Axis Distal Force Sensor for Minimally Invasive Surgical Palpation," IEEE/ASME Trans. on Mechatronics, vol. 17, no. 4, pp. 646-656, Aug. 2011.

[56] D. Zbyszewski, H. Liu, P. Puangmali, K. Althoefer, C. S. Nunes, L. D. Seneviratne, B. Challacombe, D. Murphy, and P. Dasgupta, "Wheel/tissue force interaction: a new concept for soft tissue diagnosis during MIS.," in Annual International Conference of the IEEE Engineering in Medicine and Biology Society, 2008, vol. 2008, pp. 5556-9.

[57] H. Liu, J. Li, X. Song, L. D. Seneviratne, and K. Althoefer, "Rolling Indentation Probe for Tissue Abnormality Identification During Minimally Invasive Surgery," IEEE Trans. Robot., vol. 27, no. 3, pp. 450-460, 2011.

[58] E. Knoop and J. Rossiter, "Dual-Mode Compliant Optical Tactile Sensor," in IEEE International conference on Robotics and Automation ICRA, 2013, pp. 998-1003.

[59] K. M. Chung, Z. Liu, C. Lu, and H.-Y. Tam, "Highly Sensitive Compact Force Sensor Based on Microfiber Bragg Grating," IEEE Photonics Technol. Lett., vol. 24, no. 8, pp. 700-702, Apr. 2012.

[60] U. Tan, B. Yang, R. Gullapalli, and J. P. Desai, "Triaxial MRICompatible Fiber-optic Force Sensor,” IEEE Trans. Robot., vol. 27, no. 1, pp. 65-74, 2011.

[61] R. Sargeant, H. Liu, and K. Althoefer, “An MRI Compatible Optical Multi-Axis Force/Torque Sensors Robotic Surgery," in Hamlyn Simposium 2012, 2012

[62] D. Zbyszewski, P. Polygerinos, L. D. Seneviratne, and K. Althoefer, "A novel MRI compatible air-cushion tactile sensor for Minimally Invasive Surgery," in 2009 IEEE/RSJ International Conference on Intelligent Robots and Systems, 2009, pp. 2647-2652.

[63] I. B. Wanninayake, L. D. Seneviratne, and K. Althoefer, "Novel indentation depth measuring system for stiffness characterization in soft tissue palpation," 2012 IEEE Int. Conf. Robot. Autom., pp. 46484653, May 2012.

[64] B. Darvish, S. Najarian, E. Shirzad, and R. Khodambash, "A Novel Tactile Force Probe for Tissue Stiffness Classification,” Am. J. Appl. Sci., vol. 6, no. 3, pp. 512-517, Mar. 2009.

[65] V. Jalkanen, "Hand-held resonance sensor for tissue stiffness measurements - a theoretical and experimental analysis," Meas. Sci. Technol., vol. 21, no. 5, pp. 055801-9, May 2010.

[66] [66] M. Jia, J. W. Zu, and A. Hariri, "A New Tissue Resonator Indenter Device and Reliability Study," Sensors, vol. 11, no. 1, pp. 1212-1228, Jan. 2011.

[67] M. H. Araghi and S. P. Salisbury, "A feedback based dynamic instrument for measuring Mechanical Properties of Soft Tissues for Minimally-Invasive surgery," in Smart Materials and Structures, 2009, no. October, pp. 59-69.

[68] S. Moromugi, S. Kumano, M. Ueda, and M. Q. Feng, "A sensor to measure hardness of human tissue," in IEEE Sensors, 2006, pp. 388391.
69] M. Beccani, C. Di Natali, M. E. Rentschler, and P. Valdastri, "Wireless Tissue Palpation : Proof of Concept for a Single Degree of Freedom," in IEEE International conference on Robotics and Automation ICRA, 2013, pp. 703-709.

[70] P. C. Lee, J. Turnidge, and P. J. McDonald, "Fine-Needle Aspiration Biopsy in Diagnosis of Soft Tissue Infections," J. Clin. Microbiol., vol. 22, no. 1, pp. 80-3, Jul. 1985.

[71] T. Aoki, T. Ohashi, T. Matsumoto, and M. Sato, "The pipette aspiration applied to the local stiffness measurement of soft tissues.," Ann. Biomed. Eng., vol. 25, no. 3, pp. 581-7, 1997.

[72] P. Schiavone, T. Boudou, E. Promayon, P. Perrier, and Y. Payan, "A light sterilizable pipette device for the in vivo estimation of human soft tissues constitutive laws," in 30th Annual International IEEE EMBS Conference, 2008, pp. 4298-4301.

[73] S. Diridollou, F. Patat, F. Gens, L. Vaillant, D. Black, J. M. Lagarde, Y. Gall, and M. Berson, "In vivo model of the mechanical properties of the human skin under suction.," Ski. Res. Technol., vol. 6, no. 4, pp. 214-221, Nov. 2000.

[74] M. J. Mack, "Minimally invasive cardiac surgery.," Surg. Endosc., vol. 20 Suppl 2, no. April, pp. S488-92, Apr. 2006.

[75] P. Polygerinos, A. Ataollahi, T. Schaeffter, R. Razavi, L. D. Seneviratne, and K. Althoefer, "MRI-compatible intensity-modulated force sensor for cardiac catheterization procedures.," IEEE Trans. Biomed. Eng., vol. 58, no. 3, pp. 721-6, Mar. 2011.

[76] M. C. Yip, S. G. Yuen, and R. D. Howe, "A robust uniaxial force sensor for minimally invasive surgery.," IEEE Trans. Biomed. Eng., vol. 57, no. 5, pp. 1008-11, May 2010.

[77] Y. Tanaka, K. Doumoto, A. Sano, and H. Fujimoto, “Active Tactile Sensing of Stiffness and Surface Condition Using Balloon Expansion," Sensors (Peterborough, NH), pp. 0-5, 2009.

[78] R. Ahmadi, J. Dargahi, M. Packirisamy, and R. Cecere, "A new hybrid catheter-tip tactile sensor with relative hardness measuring capability for use in catheter-based heart surgery," 2010 IEEE Sensors, pp. 1592-1595, Nov. 2010.

[79] D.-H. Kim, N. Lu, R. Ghaffari, Y.-S. Kim, S. P. Lee, L. Xu, J. Wu, R.H. Kim, J. Song, Z. Liu, J. Viventi, B. de Graff, B. Elolampi, M. Mansour, M. J. Slepian, S. Hwang, J. D. Moss, S.-M. Won, Y. Huang, B. Litt, and J. a Rogers, "Materials for multifunctional balloon catheters with capabilities in cardiac electrophysiological mapping and ablation therapy.," Nat. Mater., vol. 10, no. 4, pp. 316-23, Apr. 2011.

[80] F. M. Hendriks, D. Brokken, C. W. J. Oomens, D. L. Bader, and F. P. T. Baaijens, "The relative contributions of different skin layers to the mechanical behavior of human skin in vivo using suction experiments.," Med. Eng. Phys., vol. 28, no. 3, pp. 259-66, Apr. 2006.

[81] M. Kalantari, M. Ramezanifard, R. Ahmadi, J. Dargahi, and J. Kovecses, "Design, fabrication, and testing of a piezoresistive hardness sensor in Minimally Invasive Surgery," 2010 IEEE Haptics Symp., pp. 431-437, Mar. 2010.

[82] H. Leng and Y. Lin, "Development of a Novel Deformation-Based Tissue Softness Sensor,” Sensors (Peterborough, NH), vol. 9, no. 5, pp. 548-554, 2009.

[83] P. Peng, a S. Sezen, R. Rajamani, and a G. Erdman, "Novel MEMS stiffness sensor for in-vivo tissue characterization measurement.," in Annual International Conference of the IEEE Engineering in Medicine and Biology Society. IEEE Engineering in Medicine and Biology Society. Conference, 2009, vol. 2009, pp. 6640-3.

[84] J. W. Judy, "Microelectromechanical Systems (MEMS): Fabrication, Design and Applications," Smart Mater. Struct., vol. 10, pp. 11151134, 2001.

[85] A. Atieh, R. Ahmadi, M. Kalantari, J. Dargahi, and M. Packirisamy, "A Piezoresistive Based Tactile Sensor for Use in Minimally Invasive Surgery," in 2011 IEEE 37th Annual Northeast Bioengineering Conference (NEBEC), 2011, pp. 1-2.

[86] M. A. Qasaimeh, S. Sokhanvar, J. Dargahi, and M. Kahrizi, “A microtactile sensor for in situ tissue characterization in minimally invasive surgery.," Biomed. Microdevices, vol. 10, no. 6, pp. 823-37, Dec. 2008.

[87] J. Dargahi, S. Najarian, and M. A. Changizil, "A Novel Tactile Probe with Applications in Biomedical Robotics," in 2006 IEEE International Symposium on Industrial Electronics, 2006, pp. 33433347.

[88] S. Schostek, C.-N. Ho, D. Kalanovic, and M. O. Schurr, "Artificial Tactile Sensing in Minimally Invasive Surgery - a New Technical Approach," Minim. Invasive Ther., vol. 15, no. 5, pp. 296-304, Jan. 2006. 
[89] R. Ahmadi, M. Packirisamy, J. Dargahi, and R. Cecere, "Discretely Loaded Beam-Type Optical Fiber Tactile Sensor for Tissue Manipulation and Palpation in Minimally Invasive Robotic Surgery," Sensors (Peterborough, NH), vol. 12, no. 1, pp. 22-32, 2012.

[90] M. V. Ottermo, Ø. Stavdahl, and J. Tor A., "Palpation Instrument for Augmented Minimally Invasive Surgery," Proc. 2004 IEEE/RSJ Int. Conf. Intell. Robot. Syst., pp. 3-7, 2004.

[91] J. Dargahi, S. Najarian, and R. Ramezanifard, "Graphical display of tactile sensing data with application in minimally invasive surgery," Can. J. Electr. Comput. Eng., vol. 32, no. 3, pp. 151-155, 2007.

[92] J. Watanabe, H. Ishikawa, X. Arouette, and N. Miki, "Surface Texture and Pseudo Tactile Sensation Displayed by a MEMS-Based Tactile Display," in International conference on Intellegent Robots and Systems, 2012, vol. 7, pp. 4150-4155.

[93] F. L. H. Iii, R. K. Kramer, Q. Wan, R. D. Howe, and R. J. Wood, "Soft Tactile Sensor Arrays for Micromanipulation," in International conference on Intellegent Robots and Systems, 2012, pp. 25-32.

[94] S. Sokhanvar, M. Packirisamy, and J. Dargahi, "MEMS Endoscopic Tactile Sensor: Toward In-Situ," Sensors (Peterborough, NH), vol. 9, no. 12, pp. 1679-1687, 2009.

[95] S. P. Abbas, S. Chauhan, and S. J. Phee, "Real-time, Non-vision Sensory Feedback during Minimally Invasive Surgery," in TENCON 2009 - 2009 IEEE Region 10 Conference, 2009, pp. 1-6.

[96] M. T. Perri, A. L. Trejos, M. D. Naish, R. V Patel, and R. A. Malthaner, "New tactile sensing system for minimally invasive surgical tumour localization," Int. J. Med. Robot. Comput. Assist. Surg., vol. 6, no. March, pp. 211-220, 2010.

[97] A. Buchan, J. Bachrach, and R. S. Fearing, "Towards a Minimal Architecture for a Printable , Modular , and Robust Sensing Skin," in International conference on Intellegent Robots and Systems, 2012, pp. 33-38.

[98] M. Zillich and W. Feiten, "A versatile tactile sensor system for covering large and curved surface areas," in International conference on Intellegent Robots and Systems, 2012, pp. 20-24.

[99] M. W. Strohmayr, "The DLR Artificial Skin Step I : Uniting Sensitivity and Collision Tolerance," in IEEE International conference on Robotics and Automation ICRA, 2013, pp. 1004-1010.

[100]A. Del Prete, S. Denei, L. Natale, F. Mastrogiovanni, F. Nori, G. Cannata, and G. Metta, "Skin Spatial Calibration Using Force/Torque Measurements," in 2011 IEEE/RSJ International Conference on Intelligent Robots and Systems, 2011, pp. 3694-3700.

[101]R. Benhadj and B. Dawson, "Air Jets Imaging Tactile Sensing Device for Automation Applications," Robotica, vol. 13, no. 05, p. 521, Mar. 2009.

[102] M. Bianchi, J. C. Gwilliam, A. Degirmenci, and A. M. Okamura, "Characterization of an air jet haptic lump display.," in Annual International Conference of the IEEE Engineering in Medicine and Biology Society, 2011, vol. 2011, pp. 3467-70.

[103]N. Tanaka, M. Higashimori, and M. Kaneko, "Inverse Problem for Stiffness Sensing of Living Soft Tissue," in International Symposium on Micro-Nano Mechatronics and Human Science (MNS), 2010, pp. 89-94.

[104]T. Kawahara, Y. Miyata, K. Akayama, and M. Okajima, "Design of Noncontact Tumor Imager for Video-Assisted Thoracic Surgery," IEEE/ASME Trans. Mechatronics, vol. 15, no. 6, pp. 838-846, 2010.

[105]B. Willaert, N. Famaey, P. Verbrugghe, D. Reynaerts, and H. Van Brussel, "Design and in vivo validation of a force-measuring manipulator for MIS providing synchronized video, motion and force data," in IEEE International conference on Robotics and Automation ICRA, 2013, no. 1, pp. 4842-4847.

[106]K. A. Nichols and A. M. Okamura, "Autonomous Robotic Palpation: Machine Learning Techniques to Identify Hard Inclusions in Soft Tissues," in IEEE International conference on Robotics and Automation ICRA, 2013, pp. 4369-4374. 